\title{
Concordant Surgical Treatment: Non-melanocytic Skin Cancer of the Head and Neck
}

\author{
Wan Cheol Ryu, \\ In Chang Koh, \\ Yong Hae Lee, \\ Jong Hyun Cha, \\ Sang II Kim, \\ Chang Gyun Kim \\ Department of Plastic and Reconstructive \\ Surgery, Konyang Hospital, Konyang \\ University School of Medicine, Daejeon, \\ Korea
}

No potential conflict of interest relevant to this article was reported.

\begin{abstract}
Background: Skin cancer is the most common type of cancer. Of the 4 million skin lesions excised annually worldwide, approximately 2 million are considered cancerous. In this study, we aimed to describe a regional experience with skin cancers treated by a single senior surgeon and to provide a treatment algorithm.

Methods: The medical records of 176 patients with head and neck non-melanocytic skin cancer (NMSC) who were treated by a single surgeon at our institution between January 2010 and May 2016 were retrospectively reviewed, and their data (age, sex, pathological type, tumor location/size, treatment modality) were analyzed. Patients with cutaneous squamous cell carcinoma (cSCC) who were classified as a high-risk group for nodal metastasis underwent sentinel node mapping according to the National Comprehensive Cancer Network guidelines. Results: Among the patients with NMSC who were treated during this period, basal cell carcinoma (BCC; $n=102,57.9 \%$ ) was the most common pathological type, followed by cSCC $(n=66,37.5 \%)$. Most lesions were treated by complete excision, with tumor-free surgical margins determined via frozen section pathology. Thirty-one patients with high-metastasisrisk CSCC underwent sentinel node mapping, and 17 (54.8\%) exhibited radiologically positive sentinel nodes. Although these nodes were pathologically negative for metastasis, 2 patients $(6.5 \%)$ later developed lymph node metastases.

Conclusion: In our experience, BCC treatment should comprise wide excision with tumorfree surgical margins and proper reconstruction. In contrast, patients with CSCC should undergo lymphoscintigraphy, as nodal metastases are a possibility. Proper diagnosis and treatment could reduce the undesirably high morbidity and mortality rates.
\end{abstract}

Keywords: Skin neoplasm / Head and neck / Lymphoscintigraphy

\section{INTRODUCTION}

Currently, skin cancer accounts for approximately $23 \%-50 \%$ of all human cancers and is therefore the most common type of cancer $[1,2]$. The incidence of head and neck skin cancer is increasing steadily, and at greater rates than those of breast and thyroid cancer. Currently, head and neck non-melanocytic skin cancer (NMSC) is associated with a high curability rate (approximately $98 \%$ of diagnosed cancers) [3]. Although head and neck NMSC is associated

Correspondence: In Chang Koh

Department of Plastic and Reconstructive Surgery, Konyang Hospital, Konyang University School of Medicine, 158 Gwanjeodong-ro, Seo-gu, Daejeon 35365, Korea

E-mail: inns@naver.com

Received January 16, 2017 / Revised March 4, 2017 / Accepted March 4, 2017 with fewer metastases than other types of cancer, delayed and/or inappropriate treatment may lead to irreversible damage to the face and body or even mortality [1,4]. In this study, we aimed to describe a regional experience with NMSCs treated by a single senior surgeon. We hereby believe that we have accordingly provided useful information with which to devise a management algorithm for the treatment of these patients.

\section{METHODS}

We retrospectively reviewed the medical records of 176 patients with pathologically proven head and neck NMSC who were treated by a single senior surgeon at the Department of Plastic Surgery of 
our institute between January 2010 and May 2016. Malignant melanomas and benign skin lesions were excluded. All study participants provided written informed consent for inclusion in the study, and the study protocol received Institutional Review Board approval. Research was conducted in accordance with the 1964 Declaration of Helsinki and its later amendments. Patients' demographic data and clinicopathological features, including age, sex, tumor location and size, surgical method, pathological diagnosis, recurrence, and metastasis, were analyzed. Head and neck NMSC cases were mainly treated by complete excision, followed by frozen pathological evaluation. Reconstruction was primarily performed using local flaps to maximize the restoration of facial contours and texture (Fig. 1).

Patients with cutaneous squamous cell carcinoma (cSCC) who were classified as a high-risk group for nodal metastasis according to National Comprehensive Cancer Network guidelines [5-7] underwent sentinel node mapping. High-risk patients were defined as those exhibiting factors such as a tumor size of $>2 \mathrm{~cm}$, presence of ulceration or inflammation, chronic ulcerative or fistula lesion, and biopsy results indicating poor differentiation or neurovascular invasion $[8,9]$.

\section{RESULTS}

Our analysis included 176 patients with NMSC who were treated during the study period; most patients were treated by a single senior surgeon. Of these, 85 (48.4\%) were male and 91 (51.6\%) were female, with a male-to-female ratio of 0.94:1. The mean age was 70.8 years (range, 29-93 years). The largest group of patients ranged in age from $71-80$ years $(n=57,32.6 \%)$, followed by those aged $>80$ years $(\mathrm{n}=54,30.7 \%)$. The mean tumor size was $1.56 \pm 0.95 \mathrm{~cm}$; 149 patients had a tumor $<2 \mathrm{~cm}$ in size (T1, 84.7\%), whereas 26 had a tumor $>2 \mathrm{~cm}$ in size (T2, 15.3\%) (Table 1).

Basal cell carcinoma (BCC; $\mathrm{n}=102,57.9 \%$ ) was the most common histopathological type, followed by cSCC ( $\mathrm{n}=66,37.5 \%)$ and baso-
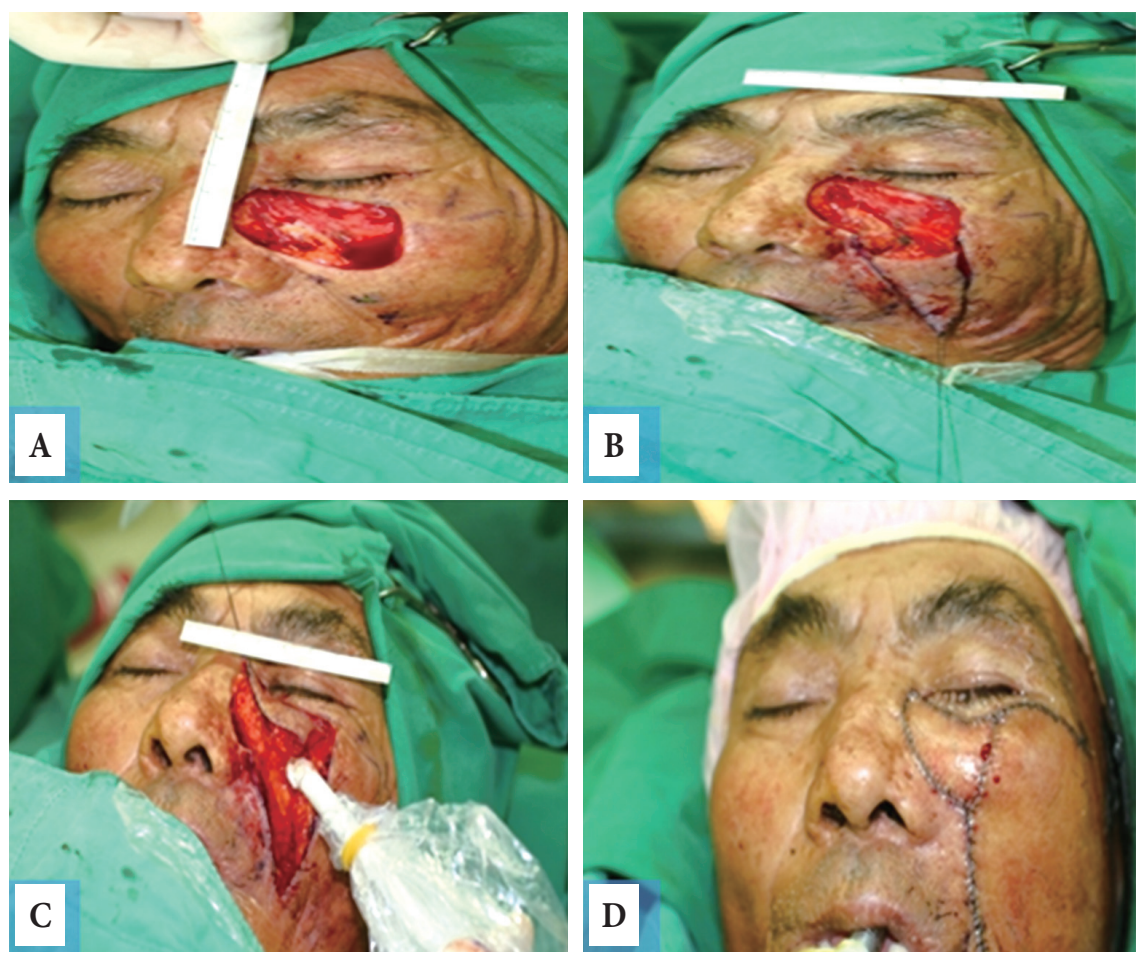

Fig. 1. Wide excision and local flap coverage (basal cell carcinoma $[\mathrm{BCC}], 1.7 \times 1.1 \mathrm{~cm}$, well-differentiated, T1N0M0, no lymphoscintigraphy). (A) Intraoperative photo 1. A large facial defect existed after wide excision. (B) Intraoperative photo 2 . The design involves an advanced local skin flap and uses an angular artery perforator. (C) Doppler evaluation of the angular artery perforator. (D) Postoperative photo. Facial reconstruction was performed via local flap coverage to maintain the facial skin color, texture, and contouring. 
squamous cell carcinoma $(\mathrm{n}=3,1.70 \%)$. Other histopathological types included microcystic adnexal carcinoma, merkel cell carcinoma, and acinic cell carcinoma of the parotid gland with skin invasion ( $n=1,0.56 \%$ for each). Six different pathological diagnoses were confirmed, and secondary tumors (i.e., metastatic carcinomas) were diagnosed in 2 patients (1.1\%) (Table 2, Fig. 2).

NMSCs were treated by complete surgical excision, followed by pathologic determination of tumor-free surgical margins. Skin defects resulting from surgical excision were reconstructed via primary closure in 19 patients (10.8\%) or covered using a local flap, fullthickness skin graft, or split-thickness skin graft in 129 (73.3\%), 18 (10.3\%), and 10 patients (5.7\%), respectively (Table 3). Thirty-one cSCC patients in the high-risk group underwent lymphoscintigraphy according to National Comprehensive Cancer Network guidelines [7]. Of these, 17 patients (54.8\%) with confirmed nodal metastases underwent regional lymph node dissection, and 15 had pathologically positive lymph nodes.

Table 1. Patient characteristics

\begin{tabular}{lccc} 
Characteristic & BCC & SCC & Patients \\
Sex & & & \\
Male & $53(51.9)$ & $24(36.4)$ & $85(48.4)$ \\
$\quad$ Female & $49(48.0)$ & $42(63.6)$ & $91(51.6)$ \\
Age (yr) & & & \\
$\quad \leq 40$ & $1(0.98)$ & 0 & $1(0.57)$ \\
$41-50$ & $6(5.88)$ & 0 & $8(4.5)$ \\
$51-60$ & $15(14.7)$ & $5(7.58)$ & $21(11.9)$ \\
$61-70$ & $28(27.4)$ & $7(10.6)$ & $35(19.9)$ \\
$71-80$ & $30(29.4)$ & $24(36.4)$ & $57(32.6)$ \\
$>80$ & $22(21.5)$ & $30(45.5)$ & $54(30.7)$ \\
Tumor size (cm) & & & \\
$\leq 2$ & $95(93.1)$ & $49(74.2)$ & $149(84.7)$ \\
$>2$ & $7(6.86)$ & $17(25.8)$ & $27(15.3)$ \\
Tumor differentiation & & & \\
$\quad$ Well & $96(94.1)$ & $3(4.54)$ & $156(88.8)$ \\
Moderate & $6(5.88)$ & $3(4.54)$ & $9(5.1)$ \\
Poor & 0 & $10(15.2)$ & $11(6.25)$ \\
Ulceration & & & \\
$\quad$ Ulcer & $3(2.94)$ & $13(19.7)$ & $16(9.1)$ \\
$\quad$ No ulcer & $99(97.1)$ & $53(83.3)$ & $160(90.9)$ \\
Neurovascular invasion & & & \\
Invasion & 0 & $5(7.58)$ & $5(2.86)$ \\
No invasion & $102(100.0)$ & $61(92.4)$ & $171(97.2)$ \\
Local recurrence & & & \\
Yes & $1(0.98)$ & $14(21.2)$ & $15(8.52)$ \\
No & $101(99.0)$ & $52(78.8)$ & $161(91.5)$ \\
Total & 102 & 66 & $176(100.0)$ \\
\hline
\end{tabular}

Values are presented as number (\%).

$\mathrm{BCC}$, basal cell carcinoma; cSCC, cutaneous squamous cell carcinoma.
Despite undergoing regional lymph node dissection, 2 patients (11.8\%) with similar cases developed recurrent nodal metastases. In one case, the patient presented with an ulcerative cancer on the lower lip, and sentinel node mapping detected positive sentinel nodes in the submandibular area via lymphoscintigraphy. The patient underwent wide excision of the lip, reconstruction with a bilateral fan flap, and regional lymph node dissection from the submandibular area. Pathological reports indicated that the submandibular nodes

Table 2. Distribution of non-melanocytic skin cancers by size

\begin{tabular}{|c|c|c|c|c|}
\hline \multirow{2}{*}{ Histopathological type } & \multicolumn{3}{|c|}{ Size (cm) } & \multirow{2}{*}{$\begin{array}{c}\text { Patients } \\
\text { (n [\%]) }\end{array}$} \\
\hline & $0-1$ & $>1, \leq 2$ & $>2$ & \\
\hline Basal cell carcinoma & 59 & 37 & 6 & $102(57.9)$ \\
\hline $\begin{array}{l}\text { Cutaneous squamous cell } \\
\text { carcinoma }\end{array}$ & 17 & 29 & 20 & $66(37.5)$ \\
\hline $\begin{array}{l}\text { Basosquamous cell } \\
\text { carcinoma }\end{array}$ & 2 & 1 & 0 & $3(1.70)$ \\
\hline $\begin{array}{l}\text { Microcystic adnexal } \\
\text { carcinoma }\end{array}$ & 0 & 0 & 1 & $1(0.56)$ \\
\hline Merkel cell carcinoma & 0 & 0 & 1 & $1(0.56)$ \\
\hline Acinic cell carcinoma & 0 & 0 & 1 & $1(0.56)$ \\
\hline $\begin{array}{l}\text { Metastatic carcinoma } \\
\text { (secondary tumor) }\end{array}$ & 0 & 0 & 2 & $2(1.1)$ \\
\hline Total & & & & $176(100.0)$ \\
\hline
\end{tabular}

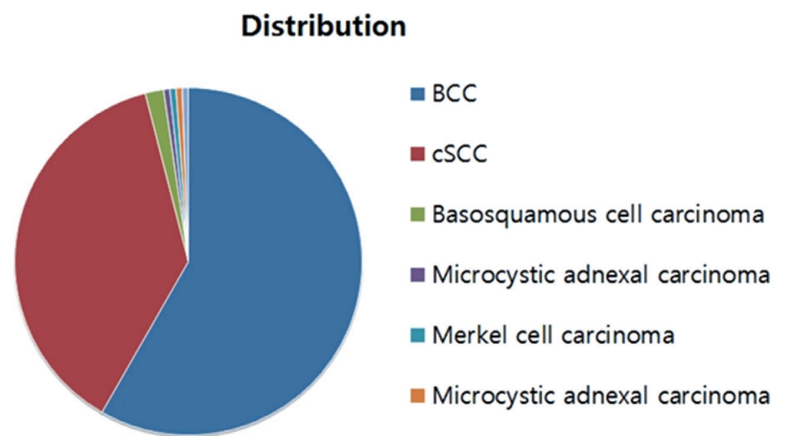

Fig. 2. Distribution of non-melanocytic skin cancers. BCC, basal cell carcinoma; cSCC, cutaneous squamous cell carcinoma.

Table 3. Analysis of coverage method frequency after wide excision

\begin{tabular}{lc} 
Coverage method & Patients \\
Primary closure & $19(10.8)$ \\
Full-thickness skin graft & $18(10.3)$ \\
Split-thickness skin graft & $10(5.7)$ \\
Local flap & $129(73.3)$ \\
Total & $176(100.0)$ \\
\hline
\end{tabular}

Values are presented as number (\%). 
were negative for cancer invasion. However, the patient developed an enlarged lymph node in the right neck after 16 months (Fig. 3).

In the other case, the patient presented with recurrent fungating mass on the lower lip, and underwent lymphoscintigraphy, during which injected dye was visualized at the right cervical level I lymph node. The patient underwent wide wedge excision of the lip, reconstruction with a modified Gillies fan flap, regional lymph node dissection, and submandibular lymph node biopsy via gamma probe.
Although pathological reports indicated that the lymph nodes were negative for malignancy, 13 months later, the patient developed an enlarged lymph node in the right neck and underwent modified radical neck dissection (Fig. 4).

Both recurrent cases underwent modified radical neck dissection by an otolaryngologist. Pathological reports indicated ipsilateral lymph node invasion with a safe margin resection. Although further evaluations (e.g., magnetic resonance imaging) have not been
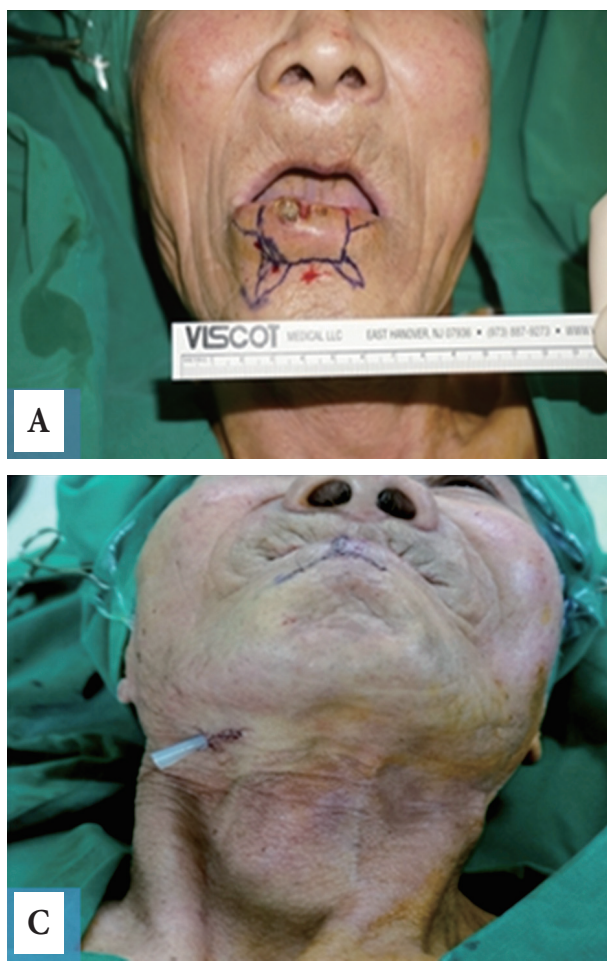

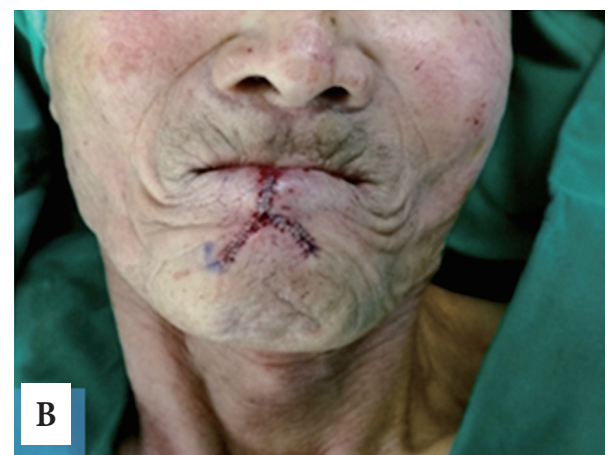

Rt

ANT

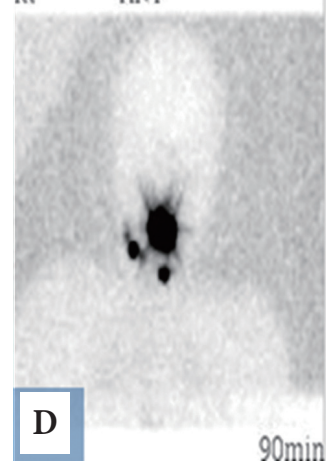

LAT

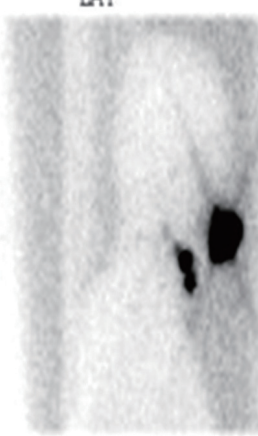

Fig. 3. Cutaneous squamous cell carcinoma of the lower lip subjected to lymphoscintigraphy $(1.2 \times 1 \mathrm{~cm}$, poorly differentiated, T1N1M0). Preoperative photo $(\mathrm{A})$ Wide excision and reconstruction of the lip were performed using a bilateral fan-like flap with sentinel node mapping and dissection. (B) Postoperative photo. (C) After regional lymph node dissection. (D) Lymphoscintigram after 90 minutes: a well-defined positive sentinel node is identified. Rt. ANT, right anterior; LAT, lateral.
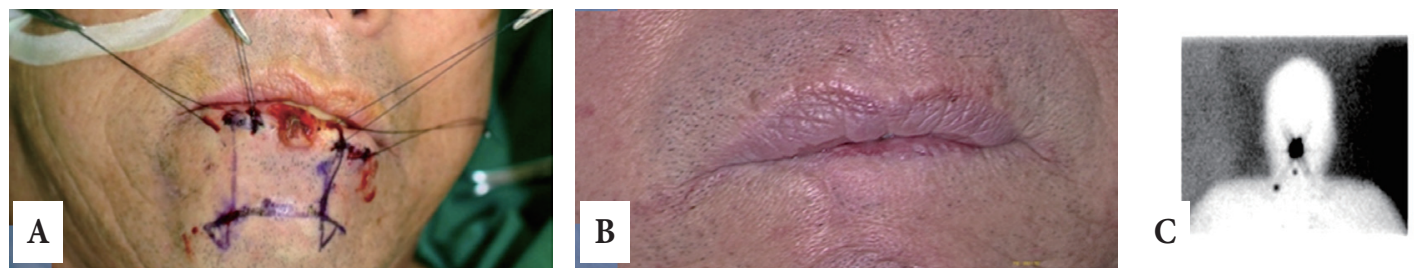

Rt LAT

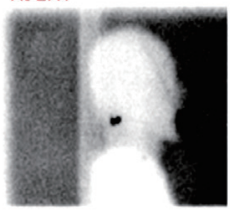

Fig. 4. Recurrent cutaneous squamous cell carcinoma of the lower lip subjected to lymphoscintigraphy $(2.1 \times 1 \mathrm{~cm}$, moderately differentiated, T2N1M0). (A) Preoperative photo. Wedge excision of the lip, reconstruction with a modified Gillies fan flap, and regional lymph node dissection with gamma probe detection were performed. (B) Postoperative photo. After 5 months, the results appeared to be aesthetically and functionally good. (C) Lymphoscintigram after 60 minutes: visualized at the right cervical level I lymph node. Rt. LAT, right lateral. 
performed, these patients have been closely followed under multidisciplinary consultation (plastic reconstructive surgery, otolaryngology, and hemato-oncology).

\section{DISCUSSION}

Skin cancer was recently reported to be the most frequently diagnosed type of cancer [1], with $>2$ million cases diagnosed annually worldwide [10]. However, skin cancer accounts for only $4 \%$ of reported malignancies in Korea, suggesting that the related statistical data are inadequate. Nevertheless, the incidence of skin cancer is increasing steadily, presumably due to changing lifestyles as well as increased exposure to ultraviolet rays, radiation, and chemical pollution; medication use; an aging population; and other environmental factors. Therefore, this disease and its diagnosis and treatment warrant additional attention [1].

BCC is the most common type of skin cancer, accounting for approximately $80 \%$ of reported cases $[11,12]$. However, BCC accounted for only $50.2 \%$ of cases at our institution. Although BCC rarely spreads to the lymph nodes or distant organs, it can invade locally and destroy neighboring tissues [13]. The most frequently affected sites include sun-exposed areas such as the face, which is subdivided into the perioral and nasal regions, upper lip, and helical portion of the ear [11]. However, BCCs have also been detected on covered areas of skin (e.g., the upper arm, chest, and thigh [13]). Treatment for this rarely metastatic cancer usually involves complete surgical excision and restoration; for the latter, the local flap method is optimal because it preserves the tissue color and texture. Skin grafts are also a useful method of restoration, although they result in a poorer aesthetic appearance and are therefore usually the last-choice method. In addition, electric desiccation, cryotherapy, 5-fluorouracil, or Mohs surgery may be used for early-stage BCC $[3,11]$. In our experience, BCCs were treated according to National Comprehensive Cancer Network guidelines [2], and 1 case of local recurrence was treated successfully by re-excision with no instance of metastasis.

cSCC is the second most common type of skin cancer, accounting for $15 \%-20 \%$ of reported cases $[9,14]$. These cancers are usually observed on sun-exposed areas such as the cheek, lower lip, perioral region, and forehead, although rarer types may arise from chronic ulcers, burns, or injuries $[1,8]$. The incidence of nodal metastasis varies, ranging from $10 \%-12 \%$ for cSCCs of the face and neck and 15\%-20\% for those arising from a chronic ulcer (Marjolin's ulcer), the lip, or around a fistula [15]. Distant metastasis with lymph node involvement can be fatal $[3,5,8,9]$. Therefore, nodal metastasis investigation requires special attention [7].

In our study, we performed both sentinel node mapping $[5,16]$ and regional lymph node dissection $[16,17]$. Despite our efforts, however, 1 of 66 patients eventually developed metastases. Patients with cSCC tumors $>2 \mathrm{~cm}$ in size with ulceration, locations surrounding fistulae, and anatomically prone locations (e.g., head and neck region cervical lymph nodes) underwent sentinel node mapping. Other malignancies, such as microcystic adnexal carcinoma, merkel cell carcinoma, and acinic cell carcinoma, were treated via complete excision with tumorfree surgical margins and follow-up. However, our sample included very few of these cases, and therefore we are unable to draw conclusions about these tumor types.

One patient with BCC of the right orbit had been treated at another institution. The disease recurred after incomplete excision, and the patient subsequently underwent radiation therapy, leading to radiation necrosis of the orbital area and subsequent blindness and necrosis of the entire right orbital region. This patient later developed SCC that eventually metastasized to the lymph nodes and lungs (Fig. 5).

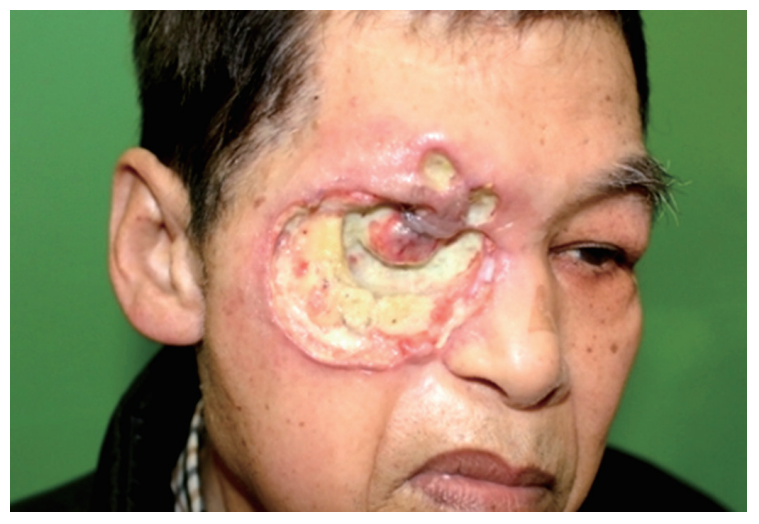

Fig. 5. Basosquamous cell carcinoma of the right orbit (poorly differentiated, T2N2M1, survival duration: 26 months). Photograph of a 67 -year-old male patient who experienced a recurrence after the incomplete surgical excision of a basosquamous cell carcinoma of the right orbit. 


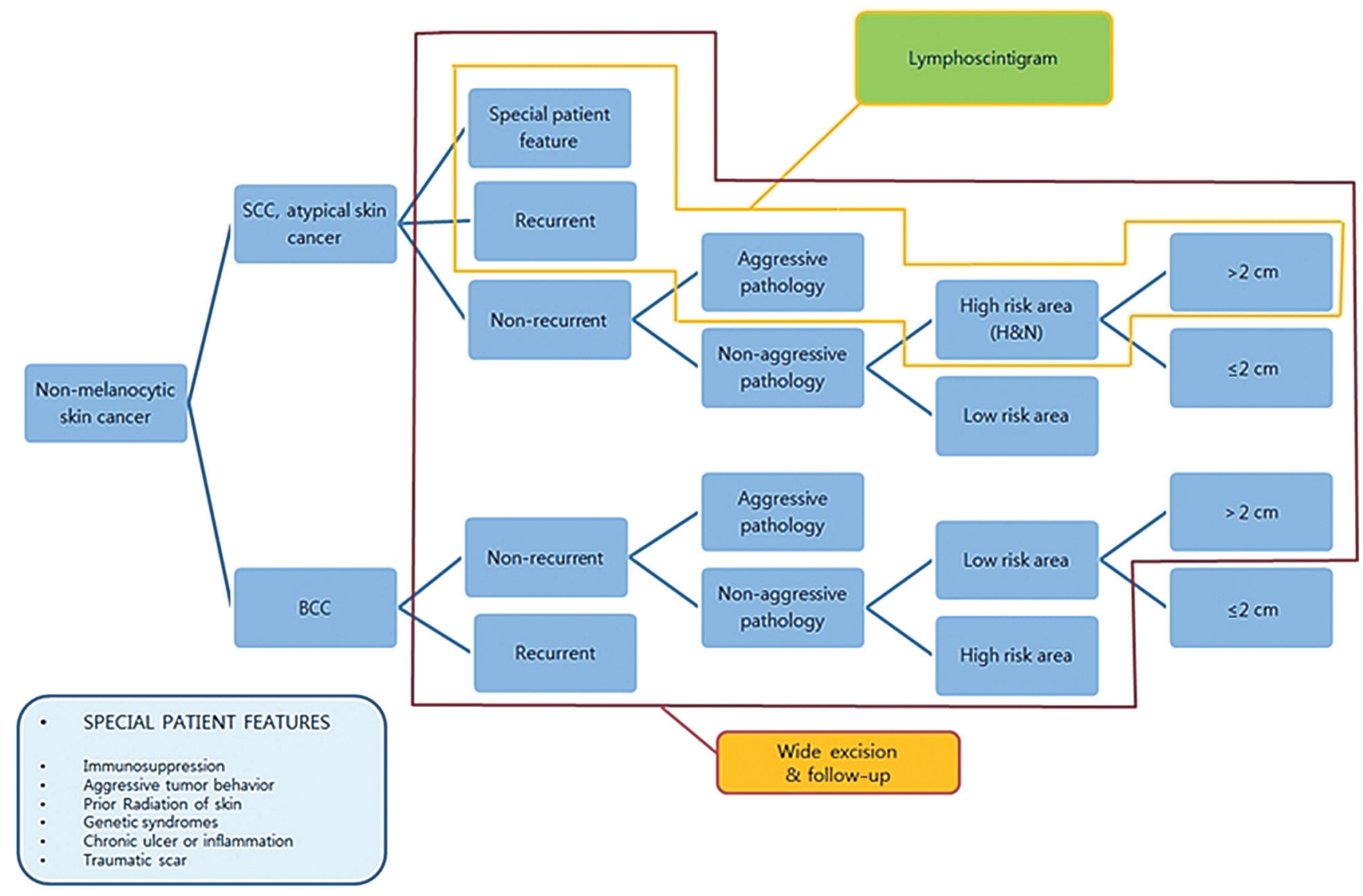

Fig. 6. Non-melanocytic skin cancer algorithm. BCC, basal cell carcinoma; cSCC, cutaneous squamous cell carcinoma; H\&N, head and neck.

As mentioned above, we experienced rarer, atypical forms of NMSC, including a case of acinic cell cancer of the parotid gland that penetrated through the skin. Although the mortality rate of NMSC is low, initial proper treatment is essential, regardless of cancer type. NMSC treatments were administered according to our institutional algorithm (Fig. 6).

In particular, the surgical application considered traditional and optimal comprised complete excision and reconstruction with a local flap to preserve the facial skin color, texture, and contour.

Although our report describes a significant investigation based on a regional experience with skin cancers treated by a single senior surgeon, we lack evidence with which to conduct a statistically significant analysis and draw conclusions regarding skin cancer treatment. It will also be necessary to evaluate the prognosis of high-risk skin cancers in a follow-up study. In future studies, we plan to identify and analyze diagnoses, treatments, and follow-up findings of high-risk cSCC patients.
In conclusion, skin cancer is presently the most common type of cancer in humans, and its incidence continues to increase. However, epidemiologic and diagnostic studies remain inadequate. Early diagnosis and appropriate treatment represent essential steps toward reducing the associated morbidity and mortality rates. In our experience, although BCCs are rarely metastatic, complete excision with tumor-free surgical margins and restoration with a local flap comprise the most effective surgical treatment. In contrast, CSCCs can cause nodal metastases that lead to devastating deformities and even death, and therefore require further nodal evaluation (e.g., lymphoscintigraphy).

\section{REFERENCES}

1. Iorio ML, Ter Louw RP, Kauffman CL, Davison SP. Evidence-based medicine: facial skin malignancy. Plast Reconstr Surg 2013;132:163143. 
2. Preston DS, Stern RS. Nonmelanoma cancers of the skin. N Engl J Med 1992;327:1649-62.

3. Kauvar AN, Arpey CJ, Hruza G, Olbricht SM, Bennett R, Mahmoud BH. Consensus for Nonmelanoma Skin Cancer Treatment, Part II: Squamous Cell Carcinoma, Including a Cost Analysis of Treatment Methods. Dermatol Surg 2015;41:1214-40.

4. Choi JH, Kim YJ, Kim H, Nam SH, Choi YW. Distribution of Basal cell carcinoma and squamous cell carcinoma by facial esthetic unit. Arch Plast Surg 2013;40:387-91.

5. Durham AB, Lowe L, Malloy KM, McHugh JB, Bradford CR, Chubb $\mathrm{H}$, et al. Sentinel Lymph Node Biopsy for Cutaneous Squamous Cell Carcinoma on the Head and Neck. JAMA Otolaryngol Head Neck Surg 2016;142:1171-6.

6. Fujimoto M, Yamamoto Y, Matsuzaki I, Warigaya K, Iwahashi Y, Kojima F, et al. Tumor budding is an independent risk factor for lymph node metastasis in cutaneous squamous cell carcinoma: a single center retrospective study. J Cutan Pathol 2016;43:766-71.

7. National Comprehensive Cancer Network. Squamous cell carcinoma NCCN clinical pracice guidelines [Internet]. Washington, PA: National Comprehensive Cancer Network; 2016 [cited 2017 Mar 11]. Available from: https:/www.nccn.org/professionals/physician_gls/f_ guidelines_nojava.asp.

8. O'Bryan K, Sherman W, Niedt GW, Taback B, Manolidis S, Wang A, et al. An evolving paradigm for the workup and management of highrisk cutaneous squamous cell carcinoma. J Am Acad Dermatol 2013;69:595-602.el.
9. Karia PS, Han J, Schmults CD. Cutaneous squamous cell carcinoma: estimated incidence of disease, nodal metastasis, and deaths from disease in the United States, 2012. J Am Acad Dermatol 2013;68:957-66.

10. Shin JH, Cho S, Whang KK, Hahm JH. An epidemiologic analysis of cutaneous malignant tumors over 15 years (1984-1998). Korean J Dermatol 1999;37:1743-51.

11. Kauvar AN, Cronin T Jr, Roenigk R, Hruza G, Bennett R. Consensus for nonmelanoma skin cancer treatment: basal cell carcinoma, including a cost analysis of treatment methods. Dermatol Surg 2015;41:55071 .

12. Ratner D. Surgical management of local basal cell carcinoma. In: Miller SJ, Maloney ME, editors. Cutaneous oncology: pathophysiology, diagnosis, and management. Malden, MA: Blackwell Science; 1998. p.664-71.

13. Alam M, Ratner D. Cutaneous squamous-cell carcinoma. N Engl J Med 2001;344:975-83.

14. Farasat S, Yu SS, Neel VA, Nehal KS, Lardaro T, Mihm MC, et al. A new American Joint Committee on Cancer staging system for cutaneous squamous cell carcinoma: creation and rationale for inclusion of tumor (T) characteristics. J Am Acad Dermatol 2011;64:1051-9.

15. Miura H, Ono S, Shibutani K, Seino H, Tsushima F, Kakehata S, et al. Contribution of dynamic sentinel lymphoscintigraphy images to the diagnosis of patients with malignant skin neoplasms in the upper and lower extremities. Springerplus 2014;3:625.

16. Parikh SA, Patel VA, Ratner D. Advances in the management of cutaneous squamous cell carcinoma. F1000Prime Rep 2014;6:70. 